\title{
A New Microstrip Filter Using CRLH Structure and Defected Ground Structure in Antenna Application
}

\author{
Xin Cao, ${ }^{1}$ Zongxi Tang, ${ }^{1}$ Fei Wang, ${ }^{2}$ Yunqiu Wu, ${ }^{1}$ and Biao Zhang ${ }^{1}$ \\ ${ }^{1}$ School of Electronic Engineering, University of Electronic Science and Technology of China, Chengdu 611731, China \\ ${ }^{2}$ Institute of Astronautics and Aeronautics, University of Electronic Science and Technology of China, Chengdu 611731, China \\ Correspondence should be addressed to Xin Cao; 798899759@qq.com
}

Received 10 December 2013; Revised 23 March 2014; Accepted 6 April 2014; Published 22 April 2014

Academic Editor: Z. N. Chen

Copyright (c) 2014 Xin Cao et al. This is an open access article distributed under the Creative Commons Attribution License, which permits unrestricted use, distribution, and reproduction in any medium, provided the original work is properly cited.

\begin{abstract}
A new microstrip bandpass filter using composite right/left-handed (CRLH) mushroom structure with interdigital capacitors and defected ground structure (DGS) is proposed. The proposed filter uses CRLH mushroom structure working at its first negative resonance mode to create the passband and DGS to increase rejection outside the passband. Simulation and measurement results are in good agreement, and low in-band insertion loss and great out-band rejection have been achieved. It is demonstrated that the combination of CRLH mushroom structure with interdigital capacitors and DGS has succeeded in achieving excellent performance. This new filter can be applied in transmitting and receiving antennas.
\end{abstract}

\section{Introduction}

In the year 1967, the Russian physicist V. Veselago had speculated a new metamaterial. He called this material "lefthanded material" because it could allow the propagation of electromagnetic waves with the electric field, the magnetic field, and the phase constant vectors building a left-handed triad, compared with conventional materials where this triad is known to be right-handed [1]. This material could exhibit many unique electromagnetic properties such as negative refractive index (NRI), reversal of Snell's law, reversal of Doppler effect, reversal of Vavilov-Čerenkov radiation, and reversal of Goos-Hänchen effect. But this material was only imaginary until it was experimentally demonstrated by the researchers Shelby et al. in 2001 [2]. In their research, they used the material that consisted of a two-dimensional array of repeated unit cells of copper strips and split ring resonators on interlocking strips of standard circuit board, and NRI was first observed. Since then, many researchers have proposed many new CLRH transmission lines and achieved various applications in the microwave field.

CRLH TLs have also been used in the filter applications. However, most reported filters employ the zeroth-order or the positive resonance modes. The negative modes, especially first negative resonance mode, are seldom discussed to design filters [3]. Moreover, the rejection outside the passband of most filters mentioned above is not so good due to the CRLH resonance properties.

In this paper, a new microstrip bandpass filter using CRLH mushroom structure with interdigital capacitors and DGS is proposed. First, the CRLH mushroom structure with interdigital capacitors has been adopted to achieve high quality factor using its first negative resonance mode. Next, a new DGS with asymmetrical split rings is utilized to create transmission zeros and increase better rejection level outside the passband. It can be seen from the fullwave simulation and measurement results that by combining these two different structures excellent performance of this proposed filter has been achieved.

\section{Theory and Design Process}

The proposed mushroom structure with interdigital capacitors is shown in Figures 1(a) and 1(b) and its equivalent circuit is shown in Figure 2. This structure consists of two interdigital capacitors at each side of the central mushroom patch to create series capacitance and the metallic via hole 


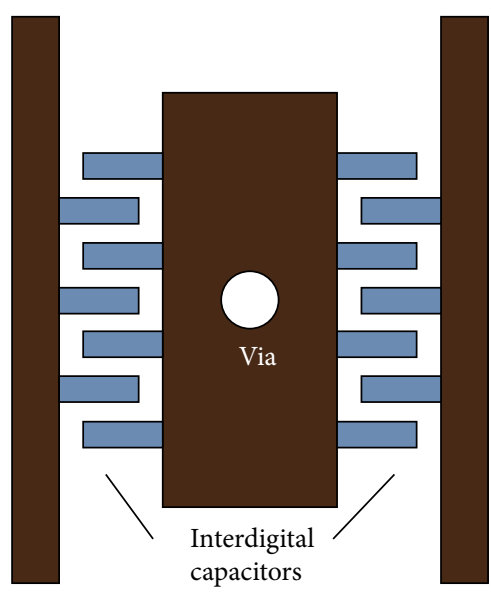

(a)

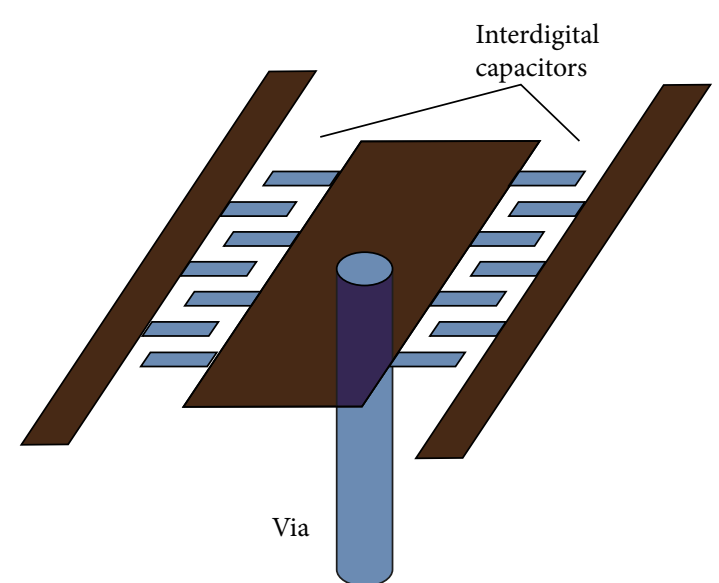

(b)

Figure 1: Top view (a) and 3D view (b) of the triangle CRLH mushroom structure.

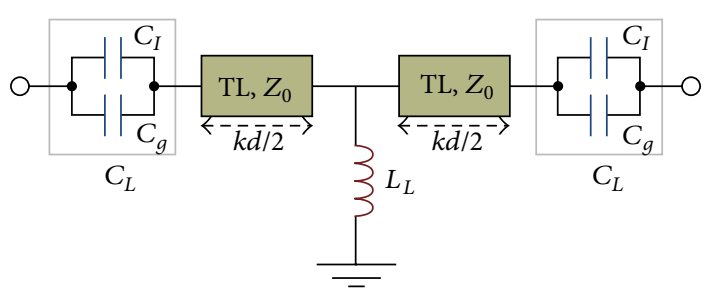

FIGURE 2: The equivalent circuit of one single mushroom structure.

in the center to create shunt inductance. And its equivalent circuit is the standard CRLH transmission line.

Based on the Bloch-Floquet theorem, the propagation constant and the normalized impedance of the CRLH TL unit cell can be expressed as [4]

$$
\begin{gathered}
\gamma(\omega)=\frac{1}{d} \cosh ^{-1}(P), \\
Z_{B}=\frac{Q}{\sqrt{P^{2}-1}},
\end{gathered}
$$

where

$$
\begin{gathered}
P=\left(1+\frac{Z Y}{4}\right) \cos \theta+\frac{j}{2}\left(Z Y_{0}+Y Z_{0}\right) \sin \theta+\frac{Z Y}{4}, \\
Q=Z \cos \theta+\frac{j}{2}\left(\frac{Z^{2} Y_{0}}{2}+Y Z Z_{0}+2 Z_{0}\right) \sin \theta \\
+\left(\frac{Z^{2} Y}{8}+\frac{Y Z_{0}^{2}}{2}\right) \cos \theta+\frac{Z^{2} Y_{0}}{8}-\frac{Y Z_{0}^{2}}{2}
\end{gathered}
$$

$Z=1 / j \omega C_{L}$, and $Y=1 / j \omega L_{L} . Z_{B}, Z_{0}$, and $Y_{0}$ are the Bloch impedance, normalized impedance, and normalized admittance of the transmission line, respectively. By using the equivalent circuit and $\mathrm{ABCD}$ matrix analysis, the dispersion equation of an infinitely long $1 \mathrm{D}$ mushroom structure can be derived as [3]

$$
\begin{aligned}
\cos \beta d= & \cos k d\left(1-\frac{1}{4 \omega^{2} L_{L} C_{L}}\right) \\
& +\sin k d\left(\frac{1}{2 \omega C_{L} Z_{0}}+\frac{Z_{0}}{2 \omega L_{L}}\right)-\frac{1}{4 \omega^{2} L_{L} C_{L}},
\end{aligned}
$$

where $k, Z_{0}$, and $d$ are the propagation constant, characteristic impedance of the transmission line, and the length of one unit cell, respectively. And the resonance condition of the CRLH TL can be derived as

$$
\beta_{n} d=\frac{n \pi d}{l}=\frac{n \pi}{M} \quad\{n=0, \pm 1, \pm 2, \ldots, \pm(M-1)\},
$$

where $d$ is the length of the unit cell and $l$ and $N(=l / d)$ are the total length of the TL and the number of the unit cells. $\beta_{n}$ is the phase constant.

The dispersion diagram is shown in Figure 3. The first negative resonance mode $(n=-1)$ is very similar to the first positive resonance mode, except that their phase constants are opposite to each other. That is, when the mushroom structure works at the first negative resonance, the electromagnetic wave is transmitted as backward wave, and when this structure works at the first positive resonance, the electromagnetic wave is transmitted as forward wave. In our design, $n=-1$ is adopted, and the first negative resonance mode has been used.

As shown in Figure 4, the defected ground structure proposed in this paper consists of a pair of asymmetrical split rings to achieve slow wave propagation and to increase the out-band rejection of the filter. The equivalent circuit is shown in Figure 5, and $C_{d n}$ and $L_{d n}$ can be calculated as

$$
\begin{gathered}
C_{d n}=\frac{5 f_{c n}}{\pi\left(f_{0 n}^{2}-f_{c n}^{2}\right)}, \\
L_{d n}=\frac{250}{\pi^{2} f_{0 n}^{2} C_{d n}},
\end{gathered}
$$




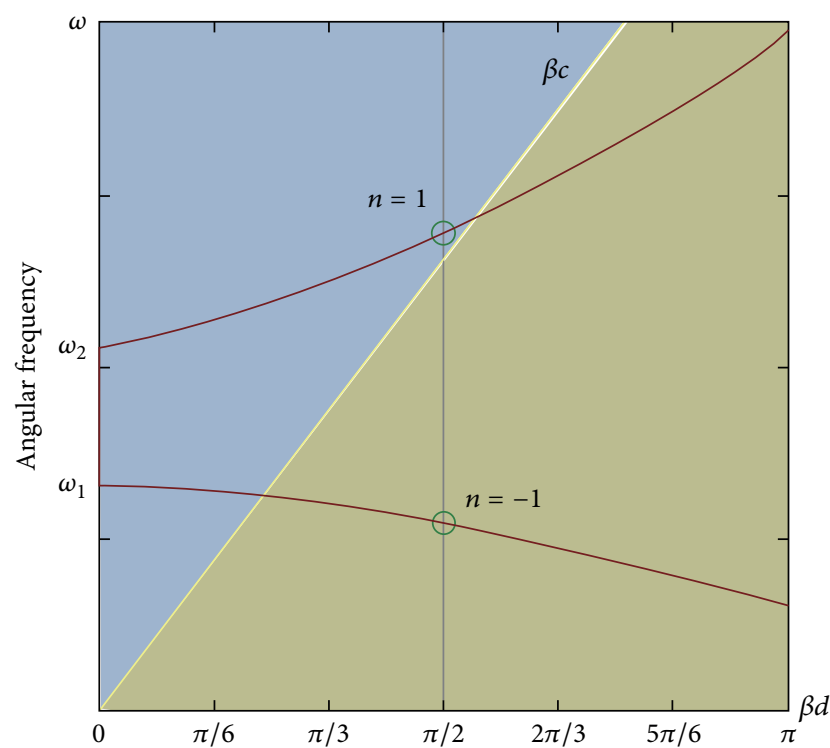

FIGURE 3: Dispersion curve of the mushroom structure.

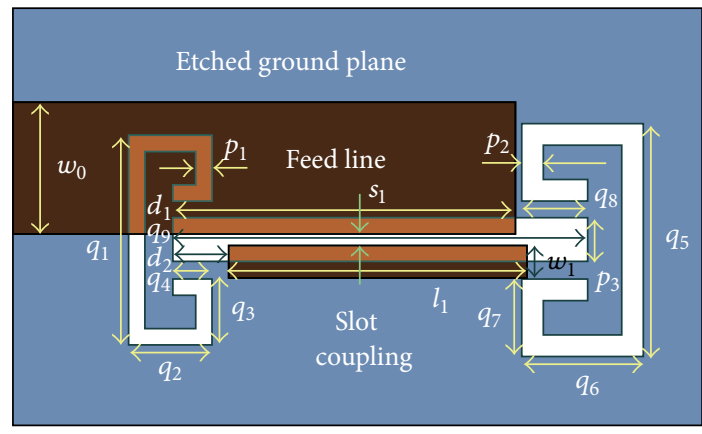

FIGURE 4: Top view of the split-ring DGS.

where $f_{c n}$ and $f_{0 n}$ are the cutoff frequencies and resonance frequencies of the stopband, and $n=1,2$.

To design the proposed filter, first, the resonance frequencies of the mushroom structure with interdigital capacitors and DGS are calculated using the formulae above based on the design specifications. The next step is tuning and optimization due to the interinfluence of the two different structures. In our design, the calculation and optimization are done mostly using Matlab and EM simulation software. After the optimization, the design process is finished.

\section{Simulation and Measurement}

The topology of the proposed filter is shown in Figure 6, and the fabricated filter is shown in Figure 7. The resonators are coupled with the input and output feed lines with the impedance of $50 \Omega$. The final simulation and measurement results are shown in Figure 8.

The measurement is done using Rohde and Schwarz's vector network analyzer. The center frequency of the passband frequency is $5.1 \mathrm{GHz}$ with the insertion loss of $1.42 \mathrm{~dB}$. This passband is produced by the first negative resonance of

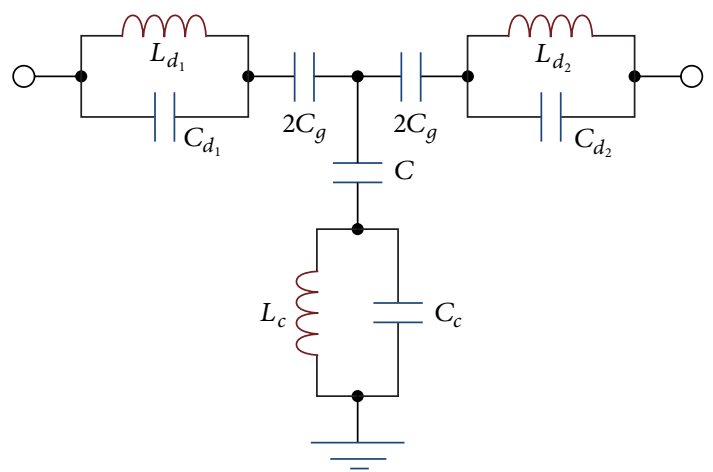

FIGURE 5: The equivalent circuit of the DGS.

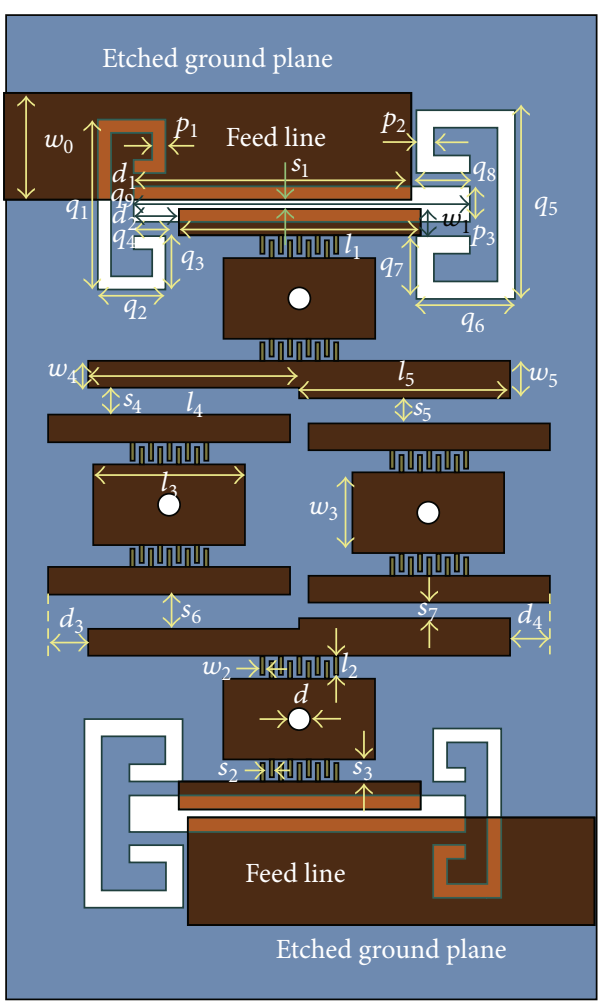

FIgURE 6: The topology of the proposed filter.

the CRLH mushroom structure. At the frequencies $4.3 \mathrm{GHz}$, $5.9 \mathrm{GHz}$, and $7.3 \mathrm{GHz}$, the DGS works to reject input signal from the feed line to enter the resonators, and, thus, transmission zeros have been created for better rejection. The filter is fabricated using the substrate Rogers 5880 with relative dielectric constant of 2.2, loss tangent $\delta=0.0009$, and thickness of $0.787 \mathrm{~mm}$. The geometric parameters are listed in Table 1 . The results have shown that the good properties of low in-band insertion loss, great out-band rejection, and compact size have been achieved and verified. The discrepancies between the simulation and measurement results are due to fabrication uncertainty.

As shown in Figure 9, in the blue region where $\beta d<0$, the curve moves from $\pi$ to 0 , and this region is the left-handed range. In the same way, in the purple region where $\beta d>0$, 
TABLE 1: Geometric parameters of the proposed filter (in mm).

\begin{tabular}{|c|c|c|c|c|c|c|c|}
\hline Parameter & Value & Parameter & Value & Parameter & Value & Parameter & Value \\
\hline$w_{0}$ & 2.4 & $w_{1}$ & 0.6 & $w_{2}$ & 0.1 & $w_{3}$ & 1.8 \\
\hline$w_{4}$ & 0.6 & $w_{5}$ & 0.8 & $l_{1}$ & 5.4 & $l_{2}$ & 0.4 \\
\hline$l_{3}$ & 3.4 & $l_{4}$ & 4.7 & $l_{5}$ & 4.7 & $s_{1}$ & 0.2 \\
\hline$s_{2}$ & 0.1 & $s_{3}$ & 0.5 & $s_{4}$ & 0.6 & $s_{5}$ & 0.6 \\
\hline$s_{6}$ & 0.8 & $s_{7}$ & 0.4 & $d_{1}$ & 6.2 & $d_{2}$ & 1.0 \\
\hline$d_{3}$ & 0.9 & $d_{4}$ & 0.9 & $p_{1}$ & 0.3 & $p_{2}$ & 0.4 \\
\hline$p_{3}$ & 0.8 & $q_{1}$ & 3.8 & $q_{2}$ & 1.5 & $q_{3}$ & 1.2 \\
\hline$q_{4}$ & 0.7 & $q_{5}$ & 4.2 & $q_{6}$ & 2.2 & $q_{7}$ & 1.4 \\
\hline$q_{8}$ & 1.2 & $q_{9}$ & 7.5 & $d$ & 0.5 & & \\
\hline
\end{tabular}

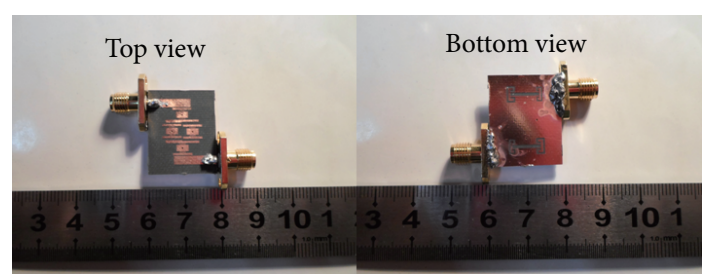

FIGURE 7: The photograph of the fabricated filter.

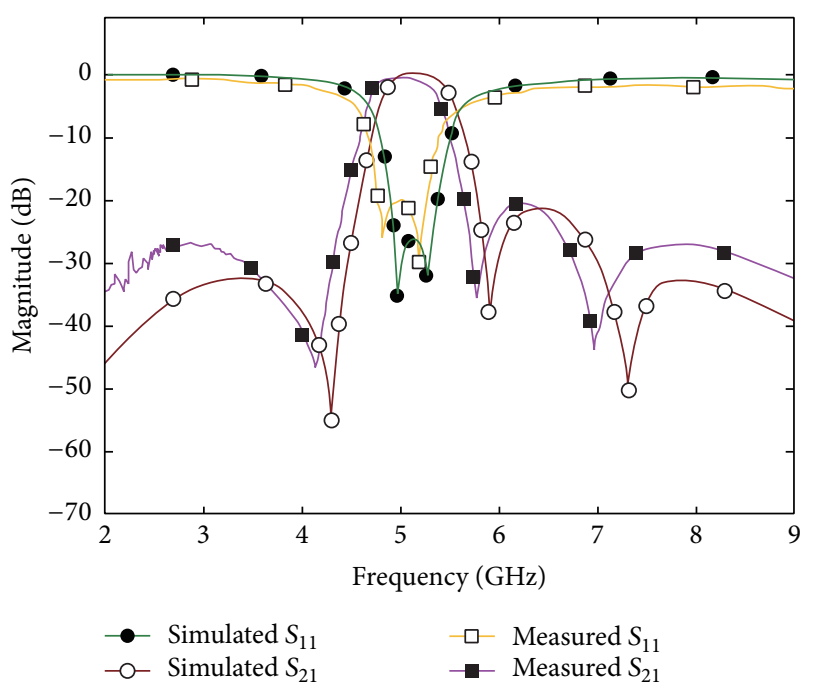

Figure 8: Simulation and measurement result.

the curve moves from 0 to $\pi$, and this region is the righthanded range. In the yellow region, $\beta d$ is around 0 , so this region is the zeroth-order resonance, which can be seen as the mixed force of left-handedness and right-handedness of the transmission line, and is the passband of the proposed filter.

\section{Conclusion}

In this paper, a new microstrip bandpass filter using CRLH mushroom structure with interdigital capacitors and DGS is proposed. The proposed filter uses CRLH mushroom structure working at its first negative resonance mode to create the passband and DGS to increase rejection outside the passband. Simulation and measurement results are in

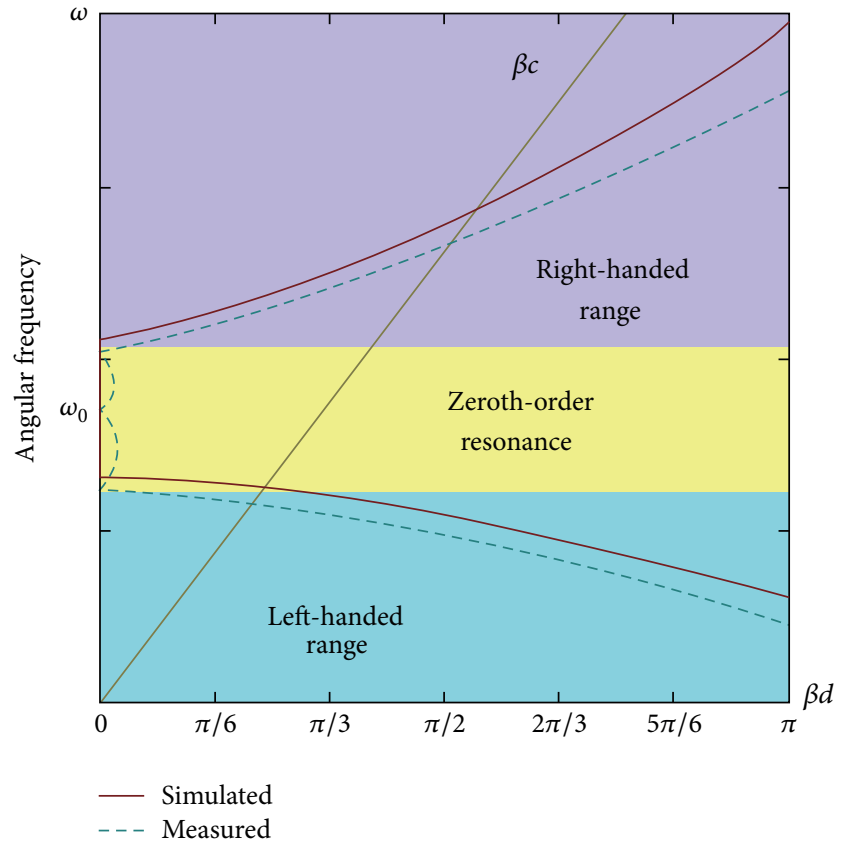

FIGURE 9: The simulated and measured dispersion curves.

good agreement and low in-band insertion loss and great outband rejection have been achieved. In real application, it is easy to redesign the filter according to new specifications. The filter is fabricated without complex fabrication process. It is demonstrated that the combination of CRLH mushroom structure with interdigital capacitors and DGS has succeeded in achieving excellent performance. This new filter can be applied in transmitting and receiving antennas.

\section{Conflict of Interests}

The authors declare that there is no conflict of interests regarding the publication of this paper.

\section{Acknowledgments}

This work is supported by the National Natural Science Foundation of China (Grant no. 61201004) and the Fundamental Research Funds for the Central Universities (ZYGX2011J011). 


\section{References}

[1] V. Veselago, "The electrodynamics of substances with simultaneously negative values of $\varepsilon$ and $\mu$," Soviet Physics Uspekhi, vol. 10, no. 4, pp. 509-514, 1968.

[2] R. A. Shelby, D. R. Smith, and S. Schultz, "Experimental verification of a negative index of refraction," Science, vol. 292, no. 5514, pp. 77-79, 2001.

[3] J.-Y. Lee, D.-J. Kim, and J.-H. Lee, "High order bandpass filter using the first negative resonant mode of composite right/lefthanded transmission line," Microwave and Optical Technology Letters, vol. 51, no. 5, pp. 1182-1185, 2009.

[4] M. Studniberg and G. V. Eleftheriades, "A quad-band bandpass filter using Negative-Refractive-Index Transmission-Line (NRI-TL) metamaterials," in Proceedings of the IEEE Antennas and Propagation Society International Symposium, pp. 49614964, June 2007. 

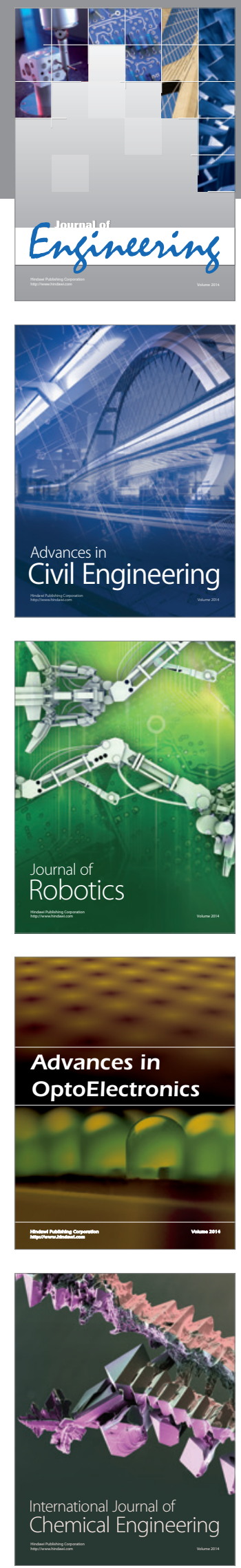

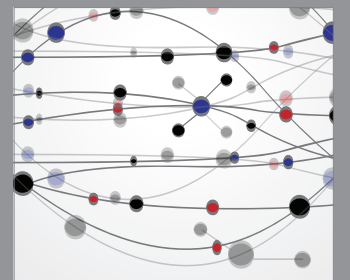

The Scientific World Journal
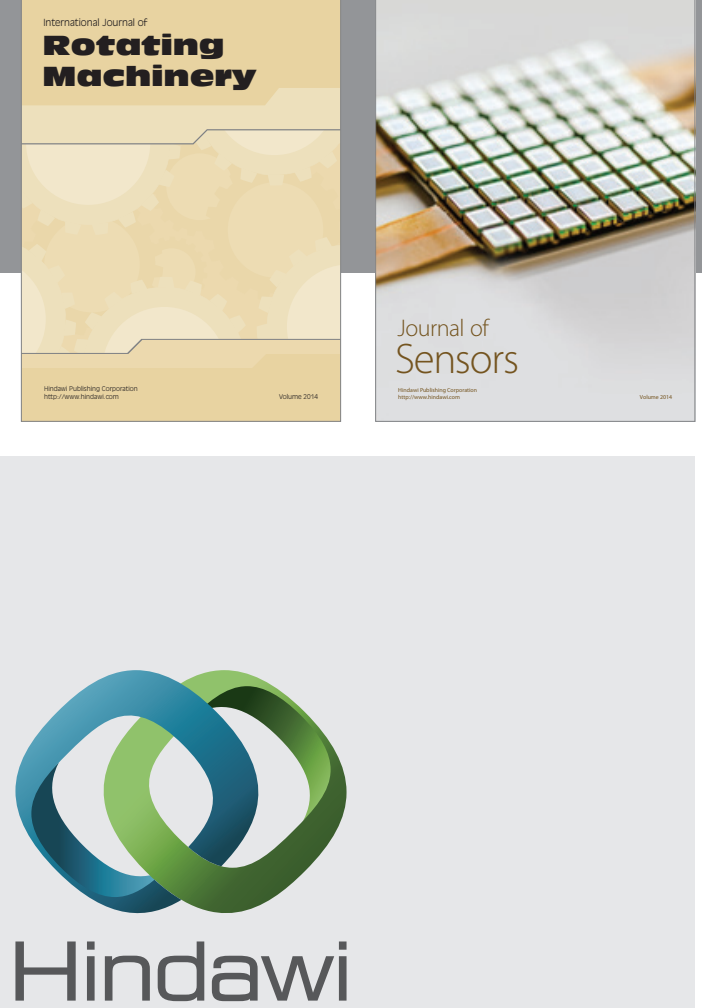

Submit your manuscripts at http://www.hindawi.com
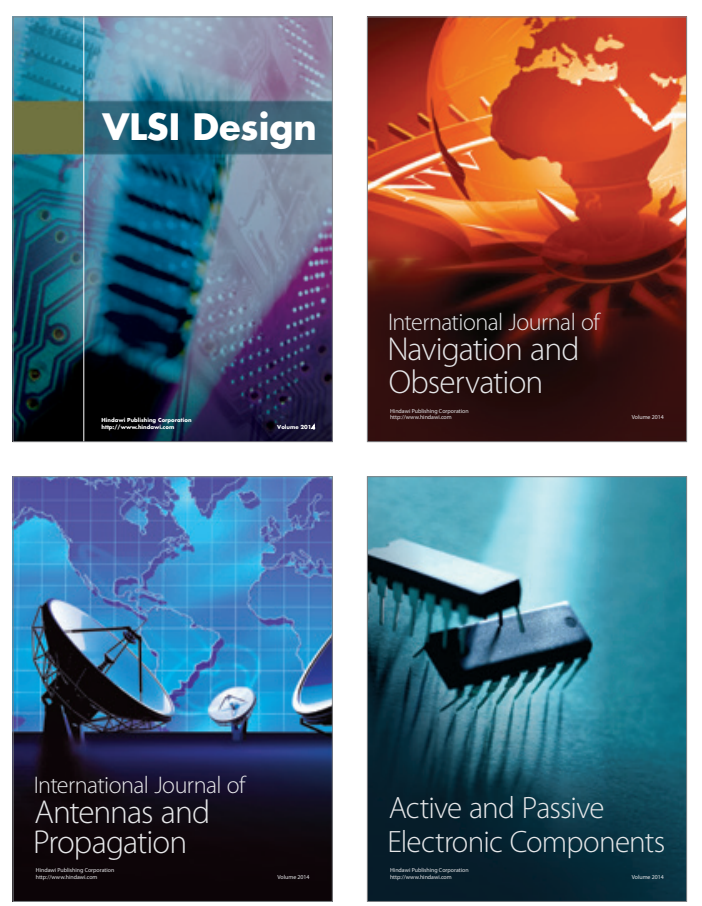
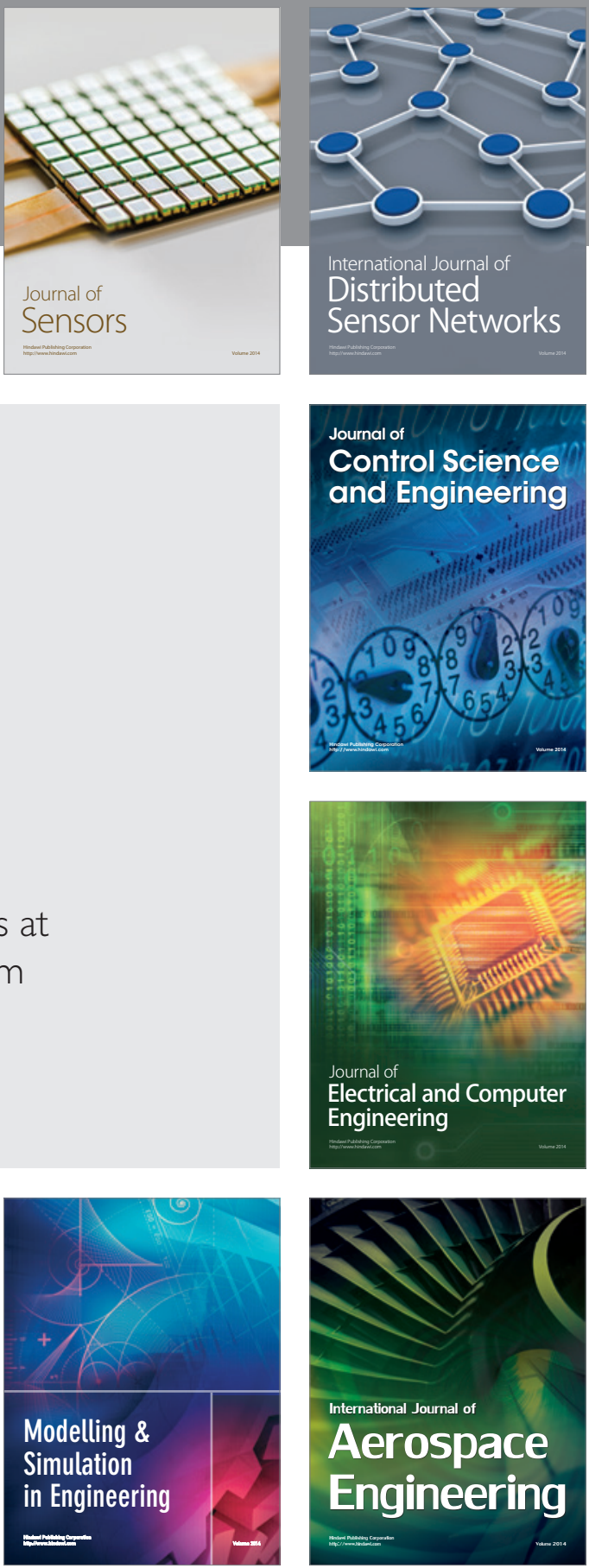

Journal of

Control Science

and Engineering
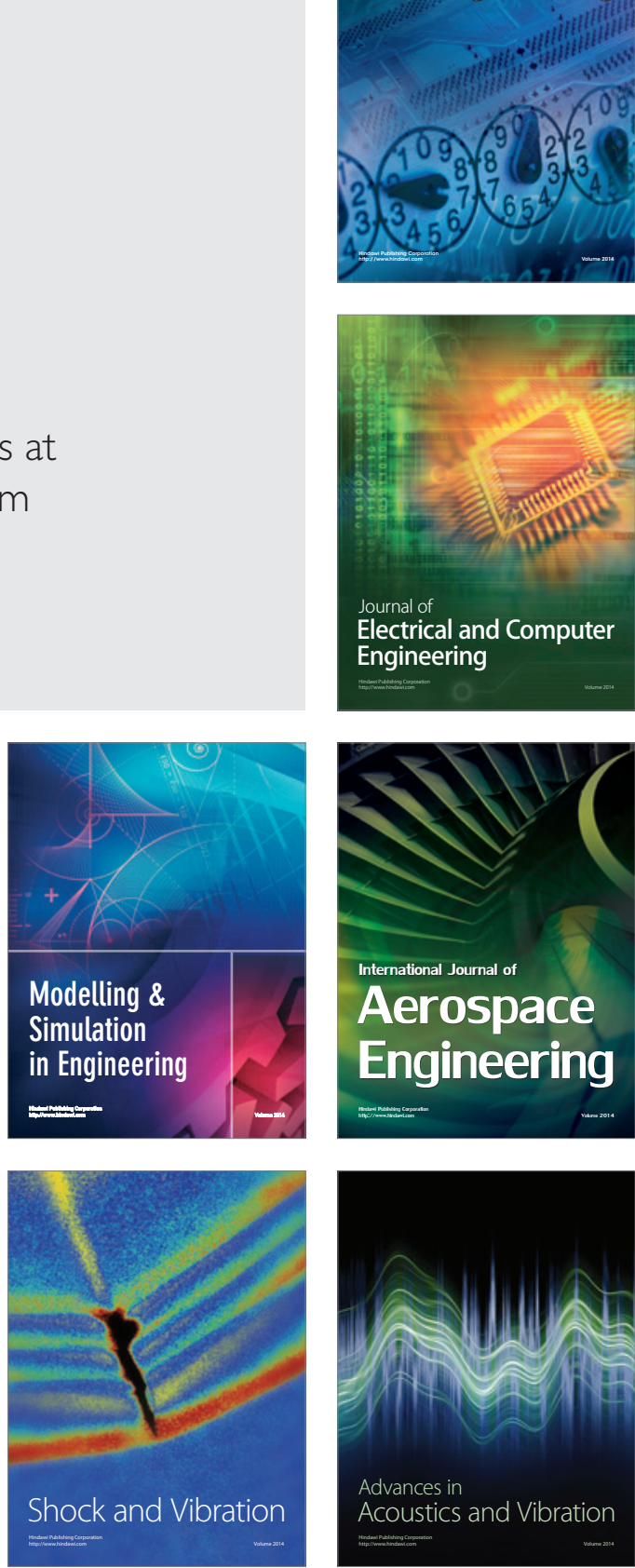